\title{
Piezo Type Mechanosensitive Ion Channel Component 1 is Required for Heart Development in Zebrafish
}

Yufeng Liu

ShanghaiTech University

\section{Zhiyu Fang}

ShanghaiTech University

Yibo Chen

ShanghaiTech University

Siyuan Zou

ShanghaiTech University

Jize Sui

ShanghaiTech University

\section{Yujia Kang}

ShanghaiTech University

jinlei shi ( $\nabla$ shijl@shanghaitech.edu.cn )

ShanghaiTech University https://orcid.org/0000-0001-9469-9614

\section{Research Article}

Keywords: PIEZO1, Zebrafish, cardiac development, RNA-seq

Posted Date: September 21st, 2021

DOl: https://doi.org/10.21203/rs.3.rs-885956/v1

License: (c) (i) This work is licensed under a Creative Commons Attribution 4.0 International License. Read Full License 


\section{Abstract}

PIEZ01 is a non-selective cation channel protein that converts mechanical stimuli into electrochemical signals through mechanical force transmission. In recent years, more and more attention has been paid to its relationship with cardiovascular development and related diseases. However, PIEZO1 is difficult to be used as a therapeutic target due to incomplete study of its related phenotype and mechanism. Starting with the phenotypes of zebrafish at different stages of development, piezo1 knockout zebrafish features decreased heart rate in embryonic stage, increased heart size in 72dpf larvae, as well as ventricular enlargement, passivation and increased immune infiltration in adult stage. Further characterization revealed the relationship between PIEZO1 and AcanA protein at embryonic stage, and hand2 protein at adult stage. Through further RNA-seq analysis, ptpn21 downregulation, shox2 upregulation, itga 4 upregulation were used to explain ventricular enlargement, heart rate decrease and immune infiltration increase respectively. Overall, this study provides a theoretical basis for elucidating the mechanism of PIEZO1 in regulating cardiac development.

\section{Introduction}

Cardiovascular disease becomes increasingly important as it contributes to high morbidity and mortality among all ages, especially for older adults. Despite the heavy burden it poses to the society, yet there is nothing like an optimal target for the disease because of its complicated mechanism[1].

The mechanically-activated (MA) cation channel protein encoded by Piezofamily is regulated by mechanotransduction. The Piezo family proteins, including PIEZO1 and PIEZO2, which participate in nonselective cationic channel activity, converse mechanical force into electrochemical signals[2].Unlike Piezo2 which exists in aspects including mechanosensory neuron, damage sensing and respiration, Piezo1 mainly distributes in non-sensory tissues, regulating osmotic homeostasis, vascular architecture, erythrocyte volume and epithelial cell division[3-7]. A wide range of researches show that PIEZO1 ion channel had important functions and its mutation related to human diseases, thus making it a considerable candidate for potential drug target[8-11]. In cardiovascular physiology, PIEZO1 is mainly supposed to be activated by the shear stress from increased blood flow or cell membrane stretch from increased blood pressure, which has close relationship with cardiovascular development and reconstitution after damage[12].

Research has shown that during early embryonic development, PIEZO1 plays an important role in vascular development, endothelial cell rearrangement and blood flow regulation[3, 12]. Piezo 1 channels are determinants of vascular structure in both development and adult physiology. Global or endothelialspecific disruption of mouse Piezo1 profoundly disturbed the developing vasculature and was embryonic lethal within days of the heart beating [3]. Studies in zebrafish have shown that under hypertension stimulation, PIEZO1 was involved in arterial remodeling process, and blocking PIEZO1-mediated signal transduction resulted in defective outflow tract and valve aortic development[13].Piezo1 controls Klf2 and Notch activity in the endothelium and Yap1 localization in the smooth muscle progenitors to coordinate 
OFT valve morphogenesis[14]. Studies in mice in which Piezo1 was conditionally deleted in endothelial cells demonstrated the requisite role of sphingosine 1-phosphate-dependent activation of Piezo1 in mediating angiogenesis in vivo[15]. Periodic stretching will induce cardiomyocyte rearrangement and PIEZ01 redistribution, and it also promote the growth of the aggregated cells expressing PIEZO1 in 3D hydrogel. Given the mechanical stimulation upon PIEZO1 inhibited, it can reduce eNOSlevel[16].

Among these current studies, although the role of PIEZO1 in the regulation of heart structure and function has been revealed, the deep mechanism of how PIEZO1 ion channel works remains unknown. There are more phenotype studies need to be carried on providing theoretical evidence for PIEZO1 as a therapeutic target. Therefore, in this study, we conduct research on piezo1 KO zebrafish about its phenotype and cardiac function over different developmental stages. Additionally, we intend to figure out PIEZO1regulated downstream genes specifically regarding cardiac functions and development, hoping to provide preliminary relationships with those phenotypes.

\section{Materials And Methods}

\section{Zebrafish line:}

The CRISPR/Cas9-mediated piezo1 ${ }^{+/-}$zebrafish line in our experiments was a gift from Professor JiulinDu[17]. The single guide RNA (sgRNA) targeting the sequence of the zebrafish piezo 1 was designed at the exon 19 , with the resulting mutation identified with a 2 bp deletion in the exon 19 , which leads to frameshift and premature stop (in the exon 20) of piezo1.

\section{Zebrafish husbandry!}

The piezo $1^{+/-}$zebrafish line and the wildtype zebrafish line were kept and raised in $10 \mathrm{~L}$ water tanks in HaiSheng aquarium (ShanghaiTech University) systems, the tanks were maintained at $26^{\circ} \mathrm{C}$ with standard photoperiod. Male and Female piezo1 ${ }^{+/-}$zebrafishes were mated to create a mixture of piezo $1^{+/+}$, piezo $1^{+/-}$, piezo $1^{-1-}$ zebrafish embryos. Embryos and larvae were raised in 1xE3 medium (5 mM $\mathrm{NaCl}, 0.17 \mathrm{mM} \mathrm{KCl}, 0.33 \mathrm{mM} \mathrm{CaCl} 2,0.33 \mathrm{mM} \mathrm{MgSO}$, 10-5\% Methylene Blue) until 8-10dpf, the fishes were transferred to the aquarium system. DNA extracts from tail fins were used to identify the genotypes of WT, piezo 1 ${ }^{+/}$, piezo $1^{1--}$ adult zebrafish, mutations of piezo 1 were examined by PCR followed by sequencing analysis with primers (Forward: 5'- ATAATATTTAAGCTGATTCATAAGCTGCAGT-3', Reverse: 5'CAGCACATGTCTTTGGACTGTGGGGGAAA-3'). Fish experiments were

compliant to the general animal welfare guidelines and protocols approved by legally authorizedanimal welfare committees (ShanghaiTech University, ShanghaiTech Animal Welfare Committee).

\section{Heart morphology and function characterization!}

Stereoscopic microscope was used to observe WT and piezo $1^{-/}$zebrafish larvae $48 \mathrm{hpf}$ and $72 \mathrm{hpf}$, the heart beats per minute of $72 \mathrm{hpf}$ zebrafishes were counted and recorded. The equipment was also used 
for heart morphology observation and heart slice HE staining observation of 7-8-month-old WT, piezo 1//, piezo $1^{-1-}$ adult zebrafish. All figures of fish larva and heart slices were observed and shot by stereoscopic microscope.

\section{Western Blotting:}

The resected hearts of WT or piezo $1^{-/}$adult zebrafish which were frozen with liquid nitrogen were lysed in RIPA (89900, Thermo Fisher) solution containing protease inhibitor cocktail (5892970001, Sigma). 4-20\% ExpressPlus $^{\text {TM }}$ PAGE Gel (M42010C, GenScript) were used for protein samples separations. Samples were transferred onto PVDF membranes (FFP26, Beyotime) at $0^{\circ} \mathrm{C}, 200 \mathrm{~mA}$ for $5 \mathrm{~h}$. Transferred membranes were blocked with 5\% non-fat milk (A600669-0250, BBI) TBST (T1081, Solarbio) solution. The following primary antibodies were used: Piezo1 (1:500, 15939-1-AP ProteinTech) and GAPDH (1:500, D1100160025, BBI). We used the following secondary antibodies: HRP-conjugated anti-rabbit secondary antibodies (1:5000, 65-6120,invitrogen) and anti-mouse secondary antibodies (1:5000, D110058-0025, $\mathrm{BBI}$ ). HRP-conjugated antibodies were detected with chemiluminescence (ECL) detection kit (ED0015-C, SparkJade).

\section{Quantitative Real time PCR (RT-qPCR):}

The total RNA from resected heart in adult or larval zebrafish was extracted with TRIzol Reagent (235002, Ambion), followed by separation with chloroform and purification with isopropanol and $70 \%$ alcohol. cDNA was synthesizedAwithPrimeScript RT Master Mix (RR036A-1, Takara) and cDNA samples were amplified with shox2, cenpf, itga4, dsc2l, tfpi2, smyd1b, uqcrb, fbxw7 andptpn21 with ChamQ Universal SYBR qPCR Master Mix (Q711-02, Vazyme). Reactions were run and analyzed with gapdh as an endogenous internal control. Primers used are listed in supplementary table 1. Relative expression values for each gene were calculated using the $\Delta \Delta \mathrm{Ct}$ analysis method.

\section{H\&E staining:}

The hearts excised from ether WT or piezo $1^{-1-}$ adult zebrafish were fixed in $10 \%$ neutral buffer formalin for at least 24h. After gradient dehydration in alcohol and infiltration in xylene, the excised tissues were embedded in paraffin and were sliced into $7-8 \mathrm{~mm}$ sections. Then the deparaffinized tissues were histologically examined following hematoxylin (E607367-0100, BBI) and eosin (E607321-0100, BBI) staining.

\section{RNA-Seq and data analysis:}

RNA was isolated following standard TRIzol protocols. After RNA extraction, the sample was shipped for library preparation.Sequencing was performed using HiSeq2500(Illumina Inc., San Diego, CA) at Genergy Biotechnology (Shanghai) Co., Ltd. Pre-alignment quality control and trimming were performed by fastp[18], removing reads less than 35 bases in length and PHREAD less than 20. Remaining reads were mapped to Danio Rerio GRCz11 reference genome using sensitive-local mode in bowtie2 
aligner[19].edgeR[20] was used to detect DE genes between control and Piezo ${ }^{\%-}$ groups. Genes were removed when they had a total read count of zero. Common dispersion was estimated by housekeeping genes from GDS3623 (DPM<0.01) as shown in supplementary table 2. Pairwise comparison between two groups showed differentially expressed genes (DEGs). Pvalues were adjusted following Benjamini\& Hochberg method, controlling the false discovery rate (FDR). Genes were regarded to be DEGs if the FDR was below 0.05 and fold change greater than 2 .

\section{Statistical analysis:}

Results were expressed as mean \pm standard deviation (SD). Mean differences between two groups were analyzed by Student's two-tailed t-test and comparisons of multiple groups were analyzed by one-way ANOVA (GraphPad; Prism) $P$ values $<0.05$ were considered significant. ${ }^{*} \mathrm{P} \leq 0.05$; ${ }^{\star *} \mathrm{P} \leq 0.01$; $* \star * \mathrm{P} \leq$ 0.001 .

\section{Result}

\subsection{Identification of PIEZO1 expression level in piezo1 KO zebrafish}

As the basis of following phenotypic experiment, we first confirmed the decrease of PIEZO1 expression level at both transcription and translation levels in homozygous piezo1 $\mathrm{KO}$ zebrafish. Furthermore, the data in transcription level from wild, heterozygous and homozygous KO strains showed that piezo1 gene has a certain quantitative effect.

\subsection{Phenotype identification of piezo1 KO zebrafish in different development level}

To compare the phenotypic changes of piezo1 KO homozygous zebrafish heart at different developmental stages, $48 \mathrm{hpf}$ and $72 \mathrm{hpf}$ fish larvae and adult fish at 7-8 month were selected for phenotypic analysis. From the perspective of morphology analysis, no sign of clear heart development abnormity was identified in fish larvae at $48 \mathrm{hpf}$ though certain individual differences were observed (Figure 4a). However, the heart of $72 \mathrm{hpf}$ fish larvae showed increased heart size and volume, shown through both the diameter and the depth of the developing heart, and with certain degrees hydropericardium. Then, we characterized $72 \mathrm{hpf}$ juvenile fish at which stage clear heartbeat can be observed and found that the heart rate of the homozygous mutants experienced a major decline (Figure 4c). Decrease of blood-pumping ability of the heart was also observed (Data not shown). The adult mutants experienced phenotypic transformation of ventricular enlargement, shown through the passivated ventricular contour and lack of apparent apex (Fig 4d), which was reminiscent of the similarly enlarged heart at $72 \mathrm{hpf}$. This morphology change was also confirmed through HE staining. Additionally, analysis of cell species showed an increase in immune cell infiltration among mutant cardiac cells (Fig 3g-3i).

3.3 Expression level of heart development key genes in piezo1 $\mathrm{KO}$ zebrafish at different development stages 
In this study, we chose genes related to the development and migration of embryonic central myocardial cell (grinch, s1p2, sdc2), genes related to the differentiation of myocardial cell (gata5, hand2), genes related to the development of artery (ace/gfg8), vein (Islet-1), arterial glands (elastin $b$ ), aortic valve (Notch1), atrioventricular valve(Ugdh), vascular endothelial cell $(F n 1, k l f 2 a)$, ventricular myosin $(v m h c)$ and extracellular matrix of cardiac cells (has2, AcanA, elastin b) [21]. All the genes were detected in wildtype zebrafish and Piezo1 KO homozygous zebrafish at different stages of development. In 48-hourembryo, the expression level of mutant AcanA Genes showed an obvious up-regulation (Fig 4a). However, the expression of level of these gene did not show any significant changes in whole adult zebrafish lysates (Supplementary Fig 1), but the expression level of hand2 gene showed a significant decrease in dissected hearts of adult zebrafish. Since hand 2 is a key factor involved in the differentiation of cardiac muscle cells, this expression will further investigate the changes in the expression level of potential upstream pathway of hand2. Previous research has shown that $n k x 2.5$ plays an important role in the signal transduction in cardiac cells, as the upstream or parallel factor of hand2[21]. Additionally, studies have shown that Hippo pathway can regulate the expression of BMP and hand2 signals through LATS1/2, thus influencing the differentiation of cardiac cells[22]. These two possible upstream pathways were selected for second sound of expression level examination, but no significant transcriptional changes were found (Fig 4c,4d).

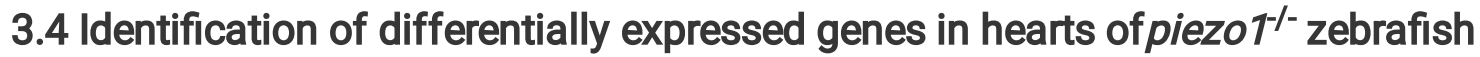

To study transcriptomic changes, piezo $1^{-1-}$ heart RNA sequencing was conducted. It was indicated that piezo1 knockout leads to 818 differentially expressed genes (DEGs), in which 535 were upregulated (Fig. $5 a)$. Among these, we concentrated on several DEGs that are related to heart functions, namely upregulated shox2, dsc2l, hbegfb, tfpi2 and downregulated smyd1b. Combined with GO annotations, 5 more genes that were not regarded as DGEs but related to heart functions were selected out (Fig. 5b), including itga4, ptpn21, cenpf, uqcrb and fbxw7. Altogether, the expression changes of these 10 genes were examined through qPCR (Fig. 5c) and showed consistent expression changes in RNA-seq data: shox2, cenpf, itga4, dsc2/ and tfpi2 were upregulated, whereas smyd1b, uqcrb, fbxw7 and ptpn 21 were downregulated. These heart-function-related genes mainly involve in 3 pathways: heart development enriched by shox2, tfpi2, dsc2l, cenpf, ptpn 21 and smyd1b, heart contraction enriched by shox2, smyd1b and $h b e g f b$, and vasculature development enriched by itga4, fbxw7, uqcrb and cenpf.

GO enrichment analysis showed top terms (Fig. 5 d, top) in biological process (phosphatidylcholine-sterol 0-acyltransferase activator activity, cholesterol binding, and cholesterol transporter activity), cellular component (nucleosome, extracellular space and extracellular region) and molecular function. To further explore the potential pathways DGEs participated in, GO enrichment analysis revealed that DGEs were also closely related to immune responses such as defense response against bacterium, innate immune response and inflammatory response, as well as cholesterol or lipid metabolism including HDL particle assembly, positive regulation of cholesterol esterification and VLDL particle remodeling (Fig. $5 \mathrm{~d}$, down).

\section{Discussion}


Piezo1 knockout caused three distinct phenotypes including reduced heart rates in $72 \mathrm{dpf}$ zebrafish larvae, heart/ventricular enlargement in $72 \mathrm{hpf}$ and adult zebrafish, and immune infiltration in adult zebrafish, suggesting defects in heart development and subsequent cardiac function.

Regarding ventricular enlargement, the downregulation of ptpn21 seemed to be relevant. Since ptpn21 morphant caused severe pericardial edema [23] in zebrafish embryo, it is possible that downregulated ptpn 21 contributes to hydropericardium as well as ventricular enlargement as a compensatory affect.To notice, there is no direct evidence reporting on the effect of long-lasting disturbance of ptpn21 and therefore the mechanism through which ventricular enlargement was caused may be novel.

For reduced heart rate, we argued that disrupted shox 2 expression may lead to developmental defects, as previous research showed the relationship between shox 2 mutant and bradycardic arrhythmia, one of whose symptoms is reduced heart rate[24]. Again, the assumption made behind this hypothesis was that the abnormal function was due to developmental failure, as the short stature homeobox 2 (shox2) transcription factor is crucial in the formation and differentiation of the sinoatrial node (SAN) [24].

Surprisingly, although PIEZO1 was reported to be essential for innate immunity, the effect of PIEZO1 on immunity in heart remains unclear. One possible explanation for the immune infiltration observed could be upregulation of itga4, which triggers homotypic aggregation for most VLA-4-positive leukocyte cell lines [25], and therefore prompts inflammatory responses.

Here, we reviewed some of the most noticeable DE genes carried out by RNA-seq. According to functional analysis, these genes were divided into three classes - heart development, heart contraction and vasculature development. Several DE genes were closely related to heart development. The late mitotic protein centromere protein $\mathrm{F}$ (cenpf) known to interact with microtubules was reported to contribute to all hallmarks of dilated cardiomyopathy (DCM) in a loss-of-function model in cardiac myocytes[26]. Previously reported depletion of tissue factor pathway inhibitor-2 (tfpi2) in zebrafish embryos caused retrenched ventricle, enlarged atrium, malformation of atrioventricular boundary and disrupted sarcomere organization [27]. dsc2/ was inferred to be an orthologue to human desmosomal cadherin desmocollin-2 [28], which was suggested as conferring susceptibility to inheritable DCM by human genetic studies [29]. The SET- and MYND domain containing protein $1 \mathrm{~b}(s m y d 1 b)$ is a member of the Smyd family that is specifically expressed in skeletal and cardiac muscles. smyd1 $b$ plays a key role in thick filament assembly during myofibrillogenesis in skeletal muscles of zebrafish embryos, knockdown of smyd1b causes significant disruption of myofibril organization in both skeletal and cardiac muscles of zebrafish embryos[30].

As for heart contraction, heparin-binding epidermal growth factor (EGF)-like growth factor (HBEGF) is a ligand for the EGF receptor (EGFR). Functional assessment in zebrafish demonstrates that when HBEGF expression is reduced with morpholino antisense reagents, it can result in a phenotype of myocardial contractile dysfunction [31]. It was reported that zebrafish Integrin alpha4 (itga4) and alpha5 function redundantly in cardiac morphogenesis and endocardial differentiation [32]. 
Lastly, we look at genes related to vasculature development. UQCRB loss of function by either genetic and pharmacological means inhibited angiogenesis [33]. F-box and WD repeat domain-containing 7 (FBXW7) is an E3-ubiquitin ligase, which serves as one of the components of the SKP1, CUL1, and F-box protein type ubiquitin ligase (SCF) complex. Previous study identified FBWX7 as an important regulator of cardiac hypertrophy [34].

Taken together, this article favors further mechanistic study on the effect of Piezo1 knockout in zebrafish.

\section{Declarations}

Conflict of interest All authors declare that they have no conflict to interest.

Consent to participate Written consent was obtained from all authors. This study was performed in strict accordance with guidelines for the care and use of laboratory animals for ShanghaiTech University. The protocols were approved by the the Shanghaitech Ethical Use of Aminals Committee (20200903003) All procedures using zebrafish were performed under Tricane anesthesia, and every effort was made to minimize discomfort and suffering.

Consent to publish All authors approved the current state of this manuscript.

Funding: This work was supported by grant from ShanghaiTech University (2021A0202-405-18).

Yufeng Liu and Zhiyu Fang have contributed equally to this work.

Author contributions LY, CY, SJ maintained the zebrafishes and performed the experiments. FZ analyzed the RNA-seq data. ZS participated in the western blot. KYJ and SJL supervised the study. All the authors wrote the manuscript.

\section{Acknowledgments}

We thank the Molecular and Cell Biology Core Facility and Zebrafish Facility at the School of Life Science and Technology, ShanghaiTech University for providing technical support. We also thank Pro.Du at Center for Excellence in Brain Science and Intelligence Technology (Institute of Neuroscience), Chinese Academy of Sciences for providing piezo1 mutant. This work was supported by grants from ShanghaiTech University (2021A0202-405-18).

\section{References}

1. van Riet, E.E., et al., Epidemiology of heart failure: the prevalence of heart failure and ventricular dysfunction in older adults over time. A systematic review. Eur J Heart Fail, 2016. 18(3): p. 242-52. DOI: 10.1002/ejhf.483.

2. Coste, B., et al., Piezo1 and Piezo2 are essential components of distinct mechanically activated cation channels. Science, 2010. 330(6000): p. 55-60. DOI: 10.1126/science.1193270. 
3. Li, J., et al., Piezo1 integration of vascular architecture with physiological force. Nature, 2014. 515(7526): p. 279-282. DOI: 10.1038/nature13701.

4. Faucherre, A., et al., Piezo1 plays a role in erythrocyte volume homeostasis. Haematologica, 2014. 99(1): p. 70-5. DOI: 10.3324/haematol.2013.086090.

5. Gudipaty, S.A., et al., Mechanical stretch triggers rapid epithelial cell division through Piezo1. Nature, 2017. 543(7643): p. 118-121. DOI: 10.1038/nature21407.

6. Kim, S.E., et al., The role of Drosophila Piezo in mechanical nociception. Nature, 2012. 483(7388): p. 209-12. DOI: 10.1038/nature10801.

7. Nonomura, K., et al., Piezo2 senses airway stretch and mediates lung inflation-induced apnoea. Nature, 2017. 541(7636): p. 176-181. DOI: 10.1038/nature20793.

8. Albuisson, J., et al., Dehydrated hereditary stomatocytosis linked to gain-of-function mutations in mechanically activated PIEZO1 ion channels. Nat Commun, 2013. 4: p. 1884. DOI: 10.1038/ncomms2899.

9. Zarychanski, R., et al., Mutations in the mechanotransduction protein PIEZO1 are associated with hereditary xerocytosis. Blood, 2012. 120(9): p. 1908-15. DOI: 10.1182/blood-2012-04-422253.

10. Lukacs, V., et al., Impaired PIEZO1 function in patients with a novel autosomal recessive congenital lymphatic dysplasia. Nat Commun, 2015. 6: p. 8329. DOI: 10.1038/ncomms9329.

11. Fotiou, E., et al., Novel mutations in PIEZO1 cause an autosomal recessive generalized lymphatic dysplasia with non-immune hydrops fetalis. Nat Commun, 2015. 6: p. 8085. DOI: 10.1038/ncomms9085.

12. Ranade, S.S., et al., Piezo1, a mechanically activated ion channel, is required for vascular development in mice. Proc Natl Acad Sci U S A, 2014. 111(28): p. 10347-52. DOI: 10.1073/pnas.1409233111.

13. Faucherre, A., et al., Piezo1 is required for outflow tract and aortic valve development. J Mol Cell Cardiol, 2020. 143: p. 51-62. DOI: 10.1016/j.yjmcc.2020.03.013.

14. Duchemin, A.L., H. Vignes, and J. Vermot, Mechanically activated piezo channels modulate outflow tract valve development through the Yap1 and KIf2-Notch signaling axis. Elife, 2019. 8. DOI: 10.7554/eLife.44706.

15. Kang, $\mathrm{H}_{\text {., }}$ et al., Piezo1 mediates angiogenesis through activation of MT1-MMP signaling. Am J Physiol Cell Physiol, 2019. 316(1): p. C92-c103. DOI: 10.1152/ajpcell.00346.2018.

16. Wong, T.Y., et al., Mechanical Stretching Simulates Cardiac Physiology and Pathology through Mechanosensor Piezo1. J Clin Med, 2018. 7(11). DOI: 10.3390/jcm7110410.

17. Liu, T.T., et al., Piezo1-Mediated Ca(2+) Activities Regulate Brain Vascular Pathfinding during Development. Neuron, 2020. 108(1): p. 180-192.e5. DOI: 10.1016/j.neuron.2020.07.025.

18. Chen, S., et al., fastp: an ultra-fast all-in-one FASTQ preprocessor. Bioinformatics, 2018. 34(17): p. i884-i890. DOI: 10.1093/bioinformatics/bty560. 
19. Langmead, B. and S.L. Salzberg, Fast gapped-read alignment with Bowtie 2. Nat Methods, 2012. 9(4): p. 357-9. DOI: 10.1038/nmeth.1923.

20. Robinson, M.D., D.J. McCarthy, and G.K. Smyth, edgeR: a Bioconductor package for differential expression analysis of digital gene expression data. Bioinformatics, 2010. 26(1): p. 139-40. DOI: 10.1093/bioinformatics/btp616.

21. Bakkers, J., Zebrafish as a model to study cardiac development and human cardiac disease. Cardiovasc Res, 2011. 91(2): p. 279-88. DOI: 10.1093/cvr/cvr098.

22. Fukui, $\mathrm{H}_{\text {., }}$ et al., Hippo signaling determines the number of venous pole cells that originate from the anterior lateral plate mesoderm in zebrafish. Elife, 2018. 7. DOI: 10.7554/eLife.29106.

23. Wyatt, L., et al., The protein tyrosine phosphatase Pez regulates TGFbeta, epithelial-mesenchymal transition, and organ development. J Cell Biol, 2007. 178(7): p. 1223-35. DOI: $10.1083 / \mathrm{jcb} .200705035$.

24. Hu, W., et al., Shox2: The Role in Differentiation and Development of Cardiac Conduction System. Tohoku J Exp Med, 2018. 244(3): p. 177-186. DOI: 10.1620/tjem.244.177.

25. Campbell, I.D. and M.J. Humphries, Integrin structure, activation, and interactions. Cold Spring Harb Perspect Biol, 2011. 3(3). DOI: 10.1101/cshperspect.a004994.

26. Manalo, A., et al., Loss of CENP-F Results in Dilated Cardiomyopathy with Severe Disruption of Cardiac Myocyte Architecture. Sci Rep, 2018. 8(1): p. 7546. DOI: 10.1038/s41598-018-25774-1.

27. Zhang, Y., et al., Tissue factor pathway inhibitor-2 is critical in zebrafish cardiogenesis. Biochem Biophys Res Commun, 2015. 456(3): p. 827-33. DOI: 10.1016/j.bbrc.2014.12.017.

28. Shih, Y.H., et al., Cardiac transcriptome and dilated cardiomyopathy genes in zebrafish. Circ Cardiovasc Genet, 2015. 8(2): p. 261-9. DOI: 10.1161/circgenetics.114.000702.

29. Hershberger, R.E., D.J. Hedges, and A. Morales, Dilated cardiomyopathy: the complexity of a diverse genetic architecture. Nat Rev Cardiol, 2013. 10(9): p. 531-47. DOI: 10.1038/nrcardio.2013.105.

30. Li, H., et al., Smyd1b is required for skeletal and cardiac muscle function in zebrafish. Mol Biol Cell, 2013. 24(22): p. 3511-21. DOI: 10.1091/mbc.E13-06-0352.

31. Friedrichs, F., et al., HBEGF, SRA1, and IK: Three cosegregating genes as determinants of cardiomyopathy. Genome Res, 2009. 19(3): p. 395-403. DOI: 10.1101/gr.076653.108.

32. Schumacher, J.A., et al., Integrin a5 and Integrin a4 cooperate to promote endocardial differentiation and heart morphogenesis. Dev Biol, 2020. 465(1): p. 46-57. DOI: 10.1016/j.ydbio.2020.06.006.

33. Cho, Y.S., et al., Functional inhibition of UQCRB suppresses angiogenesis in zebrafish. Biochem Biophys Res Commun, 2013. 433(4): p. 396-400. DOI: 10.1016/j.bbrc.2013.02.082.

34. Gao, W., et al., FBXW7 promotes pathological cardiac hypertrophy by targeting EZH2-SIX1 signaling. Exp Cell Res, 2020. 393(1): p. 112059. DOI: 10.1016/j.yexcr.2020.112059.

\section{Figures}


a

Adult Zebrafish

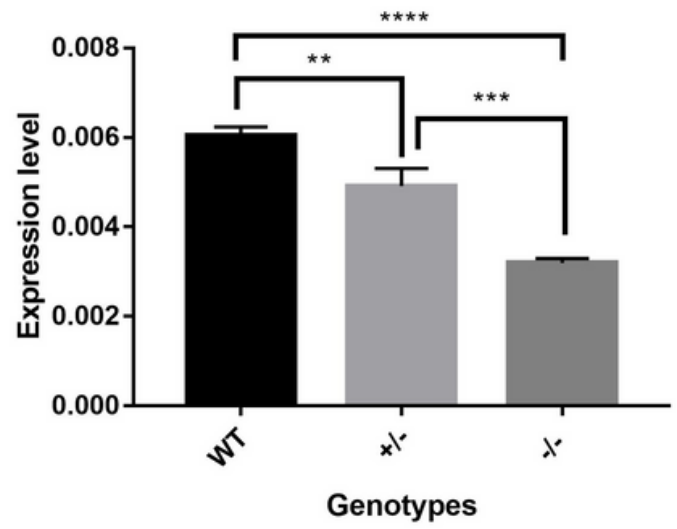

b

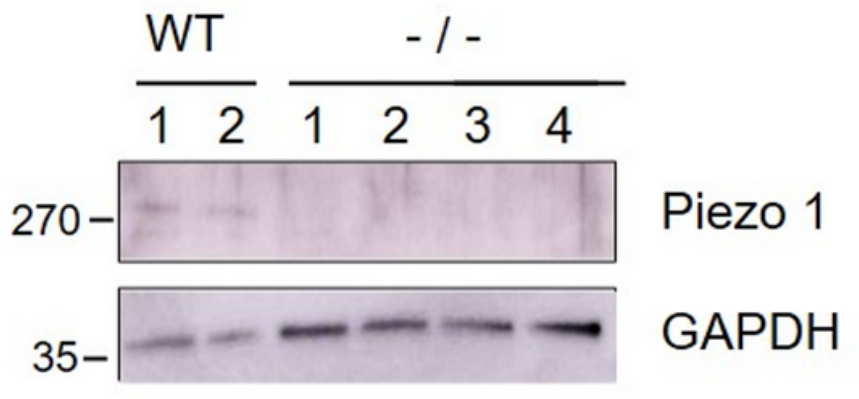

Figure 1

Caption not included with this version.

a

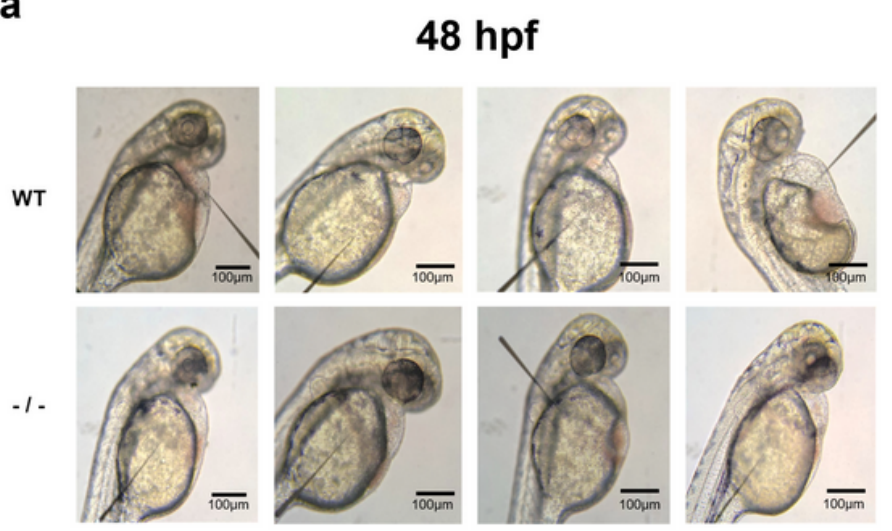

C

Heart beat per minute

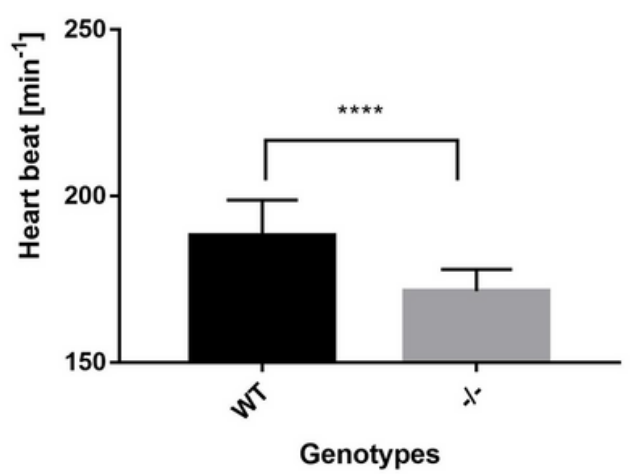

b

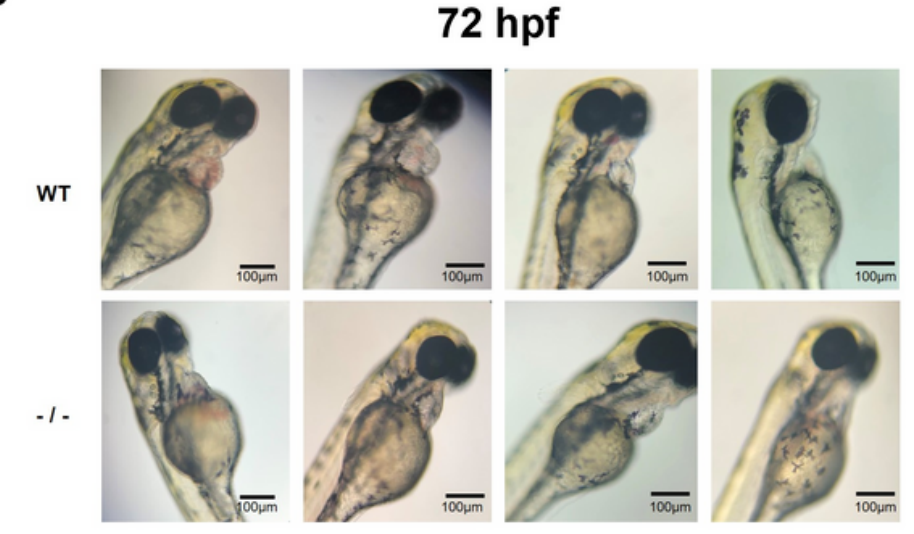

d
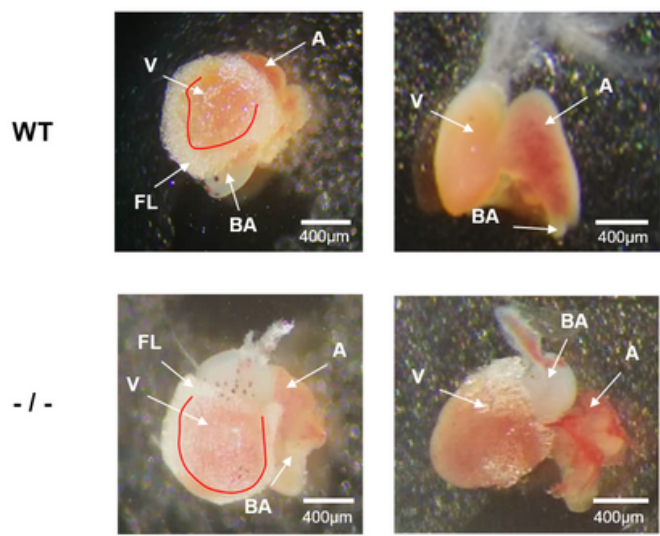

Figure 2

Caption not included with this version. 
WT
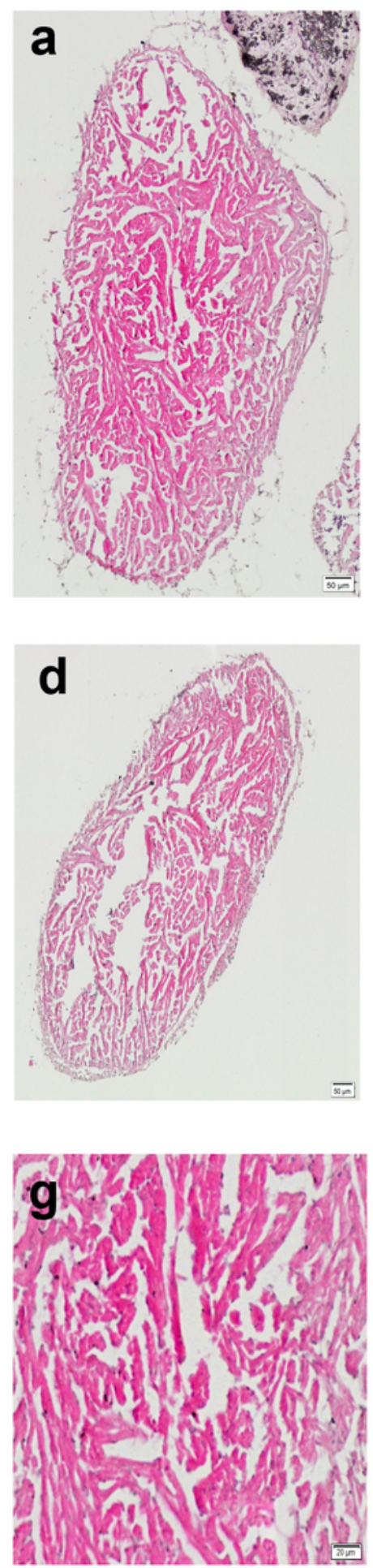

$+1-$
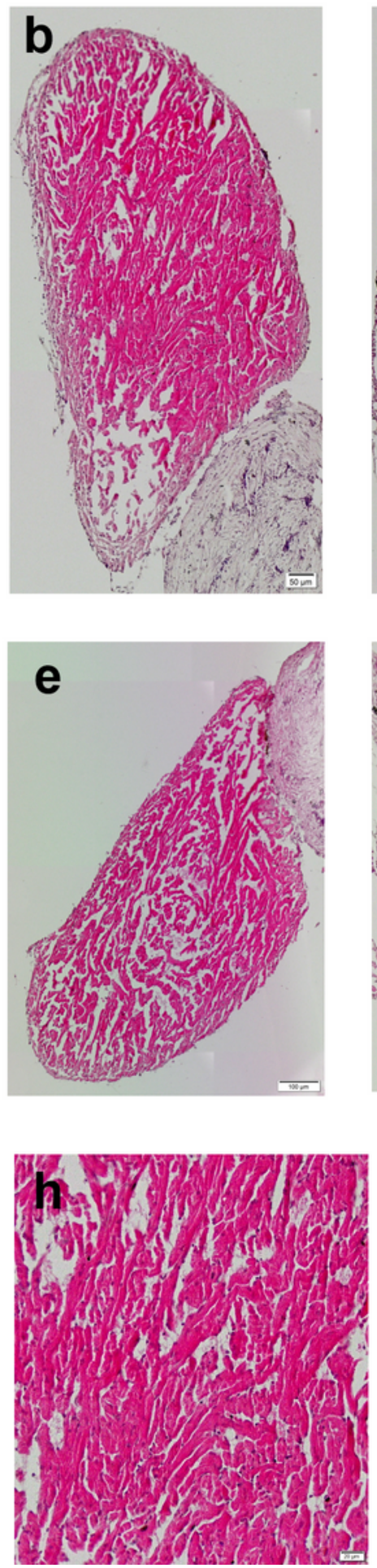
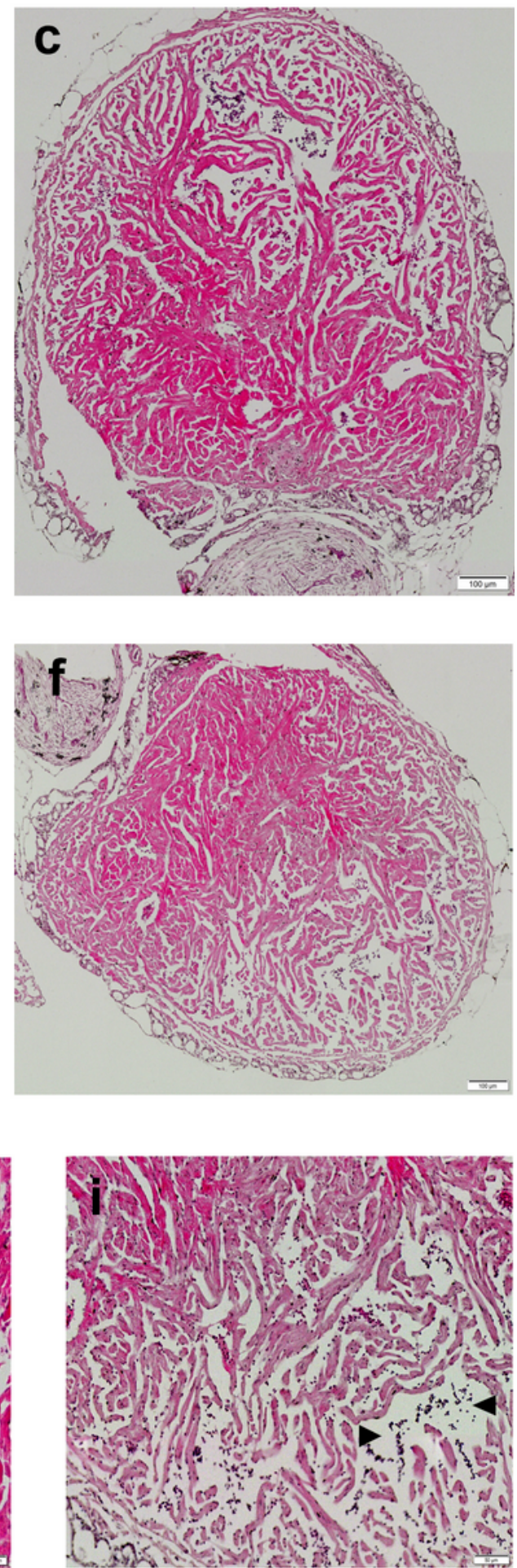

Figure 3

Caption not included with this version. 
a

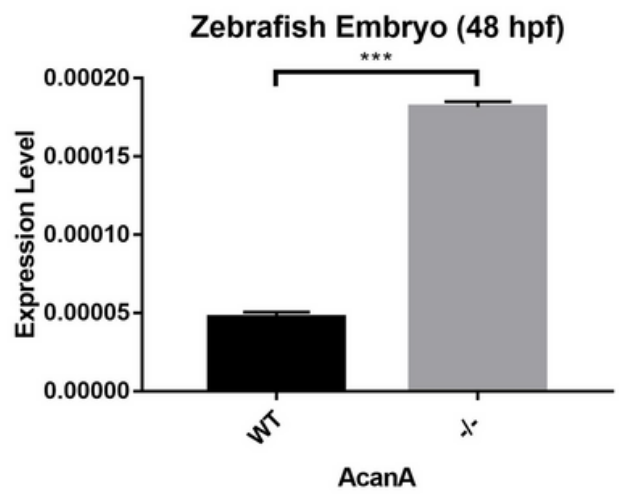

C

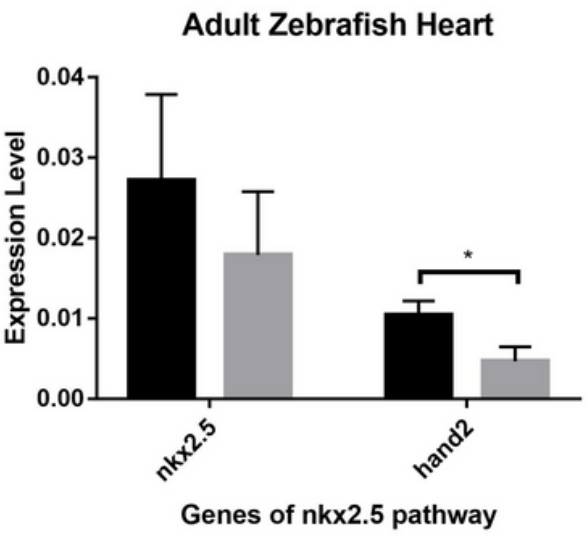

b
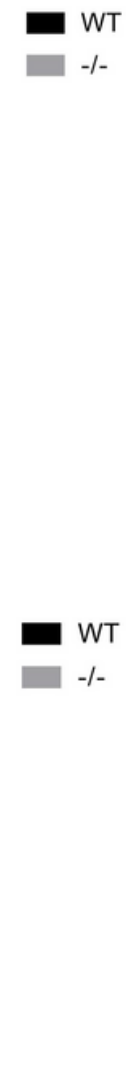

d
Adult Zebrafish Heart
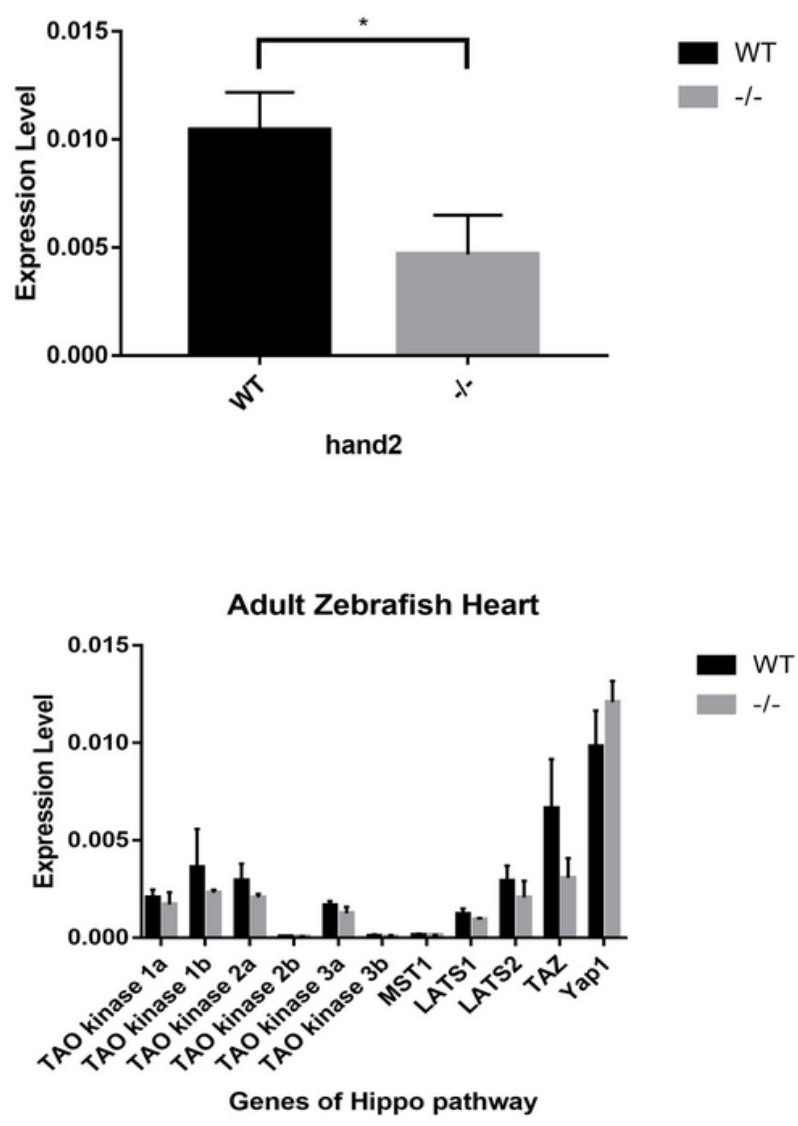

Figure 4

Caption not included with this version. 
a

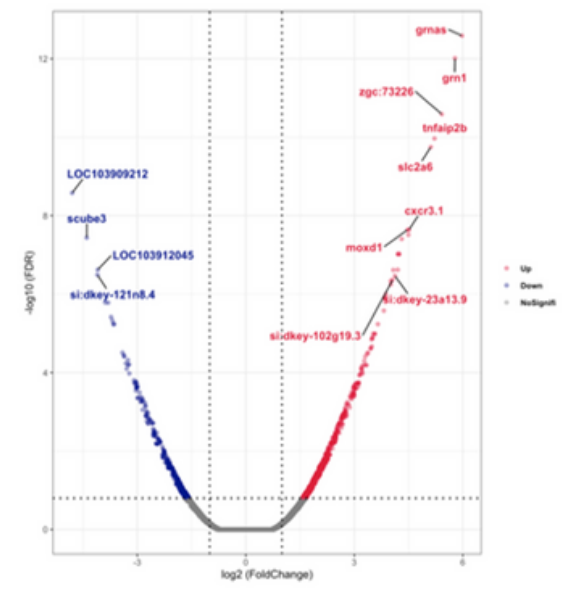

b

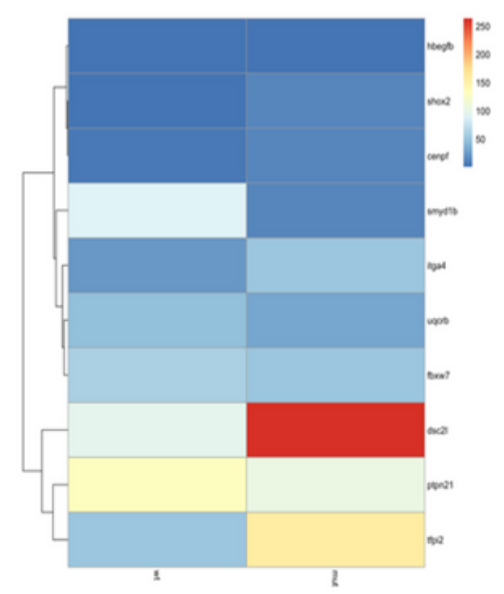

d
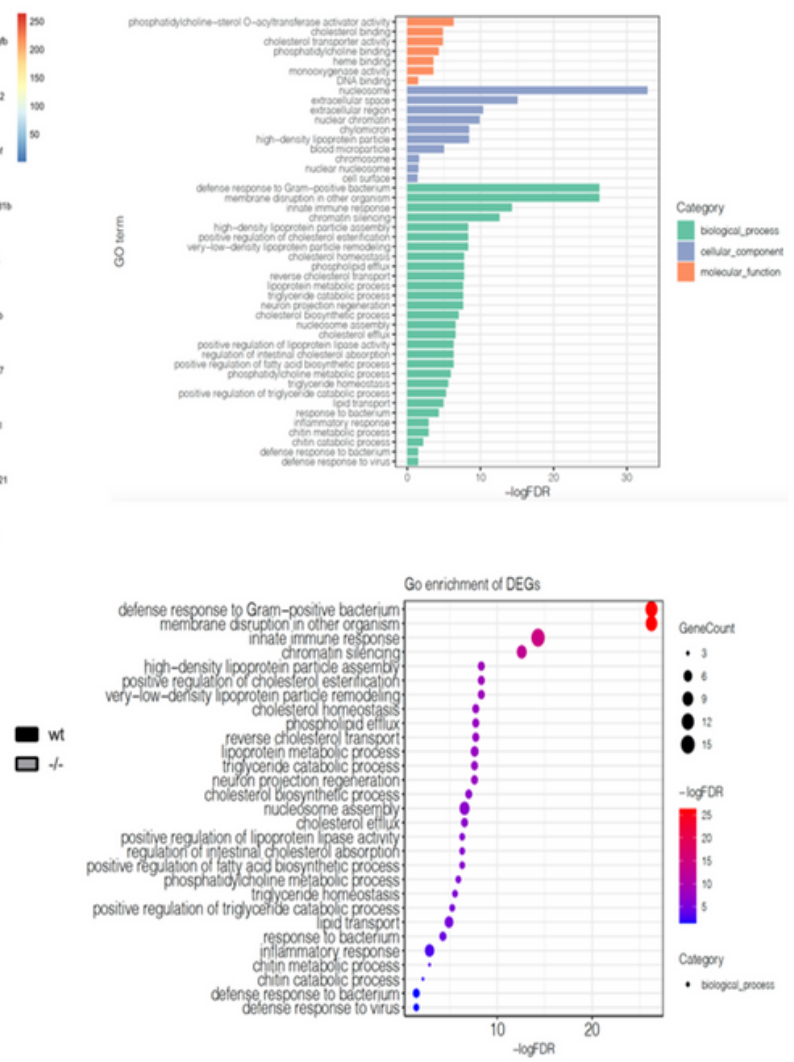

Figure 5

Caption not included with this version.

\section{Supplementary Files}

This is a list of supplementary files associated with this preprint. Click to download.

- SupplementaryFig1.jpg

- Supplementarytable2danioreriohkgenesthre001.csv

- supplementarytable1.xlsx 